\title{
Avaliação clínica de pacientes maxilectomizados pré e pós prótese obturadora: relato de experiência
}

\author{
Clinical evaluation of maxillectomized patients pre and pos filling prothesis: experience report \\ Evaluación clínica de pacientes maxilectomizados antes y después del llenado de prótesis: informe
} de experiencia

Recebido: 18/01/2021 | Revisado: 20/01/2021 | Aceito: 01/02/2021 | Publicado: 07/02/2021

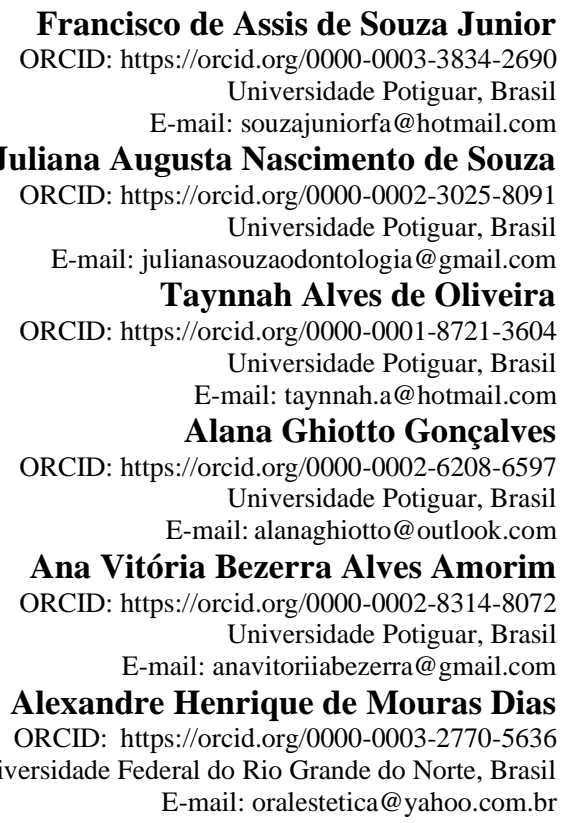

\begin{abstract}
Resumo
O uso de prótese obturadora objetiva a correção das sequelas deixada pelo procedimento cirúrgico, tem como função separar a comunicação entre a cavidade oral e as áreas superiores, protegendo o trauma ou defeito cirúrgico, contribuindo para a restauração de funções importantes para a qualidade de vida do paciente como a mastigação, deglutição e audição, além dos benefícios estéticos. Foram tratados 12 pacientes, provenientes do Hospital Dr. Luiz Antônio, que passaram por uma anamnese, exame físico extra e intra oral, responderam a questionários específicos Portuguese Brazilian, versão 3.0 (EORTC QLQC30) e Portuguese Brazilian (EORTC QLQ-H\&N35) pré e pós 15 dias da instalação protética, comparando o antes e depois através dos testes de Wilcoxon $(\alpha=0,05)$. Houve uma melhora significativa na qualidade de vida dos pacientes $(\mathrm{p}=0.009)$, nos problemas relacionados à fala $(\mathrm{p}=0.0131)$, a deglutição $(\mathrm{p}=0.0029)$ e o convívio social $(\mathrm{p}=0.0038)$. Ocorreu aumento significativo na qualidade de vida dos pacientes, redução nos problemas relacionados à deglutição, em comer socialmente e aos problemas relacionados com a fala e a maioria dos pacientes evoluíram de forma satisfatória, tendo na fala sua remissão da hipernasalidade proporcionando uma forma inteligível.
\end{abstract}

Palavras-chave: Reabilitação bucal; Qualidade de vida; Fonação; Deglutição; Audição.

\begin{abstract}
The use of obturator prosthesis aims to correct the sequelae left by the surgical procedure. Its function is to separate the communication between the oral cavity and the upper areas, protecting the trauma or surgical defect, contributing to the restoration of important functions for the quality of life of the patient. chewing, swallowing and hearing, in addition to the aesthetic benefits. 12 patients were treated, coming from Hospital Dr. Luiz Antônio, who underwent an anamnesis, extra physical examination and intra oral, answered specific questionnaires Brazilian Portuguese, version 3.0 (EORTC QLQC30) and Brazilian Portuguese (EORTC QLQ-H \& N35) pre and post 15 days of prosthetic installation, where all data were compared before and after using Wilcoxon tests $(\alpha=0.05)$. There was a significant improvement in the quality of life of patients $(\mathrm{p}=0.009)$, in problems related to speech $(\mathrm{p}=0.0131)$, swallowing $(\mathrm{p}=$ $0.0029)$ and social life $(\mathrm{p}=0.0038)$. There was a significant increase in the quality of life of patients, a reduction in
\end{abstract}


problems related to swallowing, eating socially and problems related to speech, and most patients evolved satisfactorily, having in speech their remission of hypernasality providing an intelligible form.

Keywords: Mouth rehabilitation; Quality of life; Phonation; Deglutition; Hearing.

\section{Resumen}

El uso de próteses obturatriz tiene como objetivo corregir las secuelas dejadas por el procedimento quirúrgico. Su función es separar la comunicación entre la cavidad bucal y las zonas superiores, protegendo el traumatismo o defecto quirúrgico, contribuyendo a la restauración de funciones importantes para la calidad de vida del paciente. Masticar, tragar y oír, además de los benefícios estéticos. Se atendieron 12 pacientes, provenientes del Hospital Dr. Luiz Antônio, a quienes se les realizo anamnesis, exploración física extra e intraoral, respondieron cuestionarios específicos Portugués Brasileño, versión 3.0 (EORTC QLQC30) y Portugués Brasileño (EORTC QLQ-H \& N35) pre y después de 15 dias de la instalación de la próteses, donde se compararon todos los datos antes y después de usar las pruebas de Wilcoxon $(\alpha=0.05)$. Hubo una mejora significativa en la calidad de vida de los pacientes $(\mathrm{p}=0,009)$, en los problemas relacionados con el habla $(\mathrm{p}=0,0131)$, la deglución $(\mathrm{p}=0,0029)$ y la vida social $(\mathrm{p}=0,0038)$. Hubo un aumento significativo en la calidad de vida de los pacientes, una reducción de los problemas relacionados con el habla, y la mayoría de los pacientes evolucionaron satisfactoriamente, teniendo en el habla su remisión de hipernasalidad proporcioando una forma inteligible.

Palabras clave: Rehabilitación bucal; Calidad de vida; Fonación; Deglución; Audición.

\section{Introdução}

O câncer é considerado um problema global de saúde que tende a aumentar, especialmente num mundo em desenvolvimento. Conforme o INCA as estimativas para os anos de 2020-22, é de 15.210 casos novos de câncer. A epidemiologia do câncer da boca é considerada um problema global de saúde, especialmente num mundo em desenvolvimento. As incidências regionais e ao redor do mundo estão relacionadas aos dois principais fatores: tabagismo e ingestão de bebidas alcoólicas. O câncer de boca é um tumor maligno que afeta toda a cavidade oral (Lábios, estruturas da boca, como gengivas, bochechas, céu da boca, língua principalmente as bordas e a região embaixo da língua), tem maior incidência entre pacientes com idade igual ou superior a 40 anos, configurando-se como o quinto tumor mais frequente entre o sexo masculino, além disso estima-se cerca de 15.210, sendo 11.200 homens e 4.010 mulheres (INCA - 2020). (Torres-Pereira et al., 2012; INCA, 2020)

Etiologicamente o câncer de boca tem outros fatores além da idade e gênero do indivíduo, dentre eles pode-se destacar o tabagismo, o consumo exagerado de álcool, exposição a produtos químicos e radiação solar, infecções provocadas pelo HPV, (papilomavírus humano) estão ligadas ao desenvolvimento de câncer. (Elrefaey et al., 2014)

A maioria dos casos de câncer são diagnosticados de forma tardia o que compromete o tratamento e prognóstico e a sobrevida dos pacientes assim sendo estima-se aproximadamente cerca de $50 \%$ tem sobrevida de 5 anos em casos de carcinomas orais e orofaríngeo (Epstein et al., 2008; Martínez, Hernández, Martinez, \& Adorno, 2016).

O tratamento cirúrgico do câncer tem dois objetivos principais: ressecção do tumor e de outros tecidos envolvidos, como gânglios linfáticos, e para remover órgãos endócrinos que podem alterar e expandir a doença. Diferente da cirurgia, a radioterapia e a quimioterapia não atuam em tecidos específicos; agem inibindo o crescimento celular, interferindo no mecanismo de divisão celular. Sendo assim, não diferenciam as células cancerosas das células normais. As perdas provocadas pela cirurgia de remoção desses tumores geralmente são significativas, atingem o nível superior da mandíbula, seio maxilar e passagens nasais, estabelecendo uma comunicação anormal entre a cavidade oral e anexada, mesmo que o tratamento ofereça cura ao paciente deixam sequelas e mutilação que atinge a vida do indivíduo desde funções como a comunicação, deglutição e estética (de Lima et al., 2015).

A odontologia tem se preocupado em dar sua contribuição no acompanhamento do câncer bucal, além da forma preventiva nos diagnósticos e tratamento das lesões, mas também na reparação dos defeitos dos maxilares provenientes dos tratamentos das neoplasias malignas. Por consequência é essencial essa correção, seja ela cirúrgica ou protética. O uso de 
prótese obturadora objetiva a correção das sequelas deixada pelo procedimento cirúrgico, tem como função separar a comunicação entre a cavidade oral e as áreas superiores, protegendo o trauma ou defeito cirúrgico, contribuindo para a restauração de funções importantes para a qualidade de vida do paciente como a mastigação, deglutição audição, além dos benefícios estéticos. Essa primeira opção é uma abordagem altamente positiva e não invasiva além de oferecer a possibilidade de reabilitação imediata. (de Lima et al., 2015; Miracca, Andrade Sobrinho, \& Gonçalves, 2007; Goiato et al., 2006)

Mediante as sequelas mencionadas acima, verifica-se a importância de uma avaliação protética do grau de eficácia das próteses obturadoras na qualidade de vida dos pacientes maxilectomizados através da avaliação pré e pós-prótese obturadora (deglutição, melhora das funções mastigatórias, avaliação da inteligibilidade da fala), essencial para a recuperação da auto-estima.

\section{Metodologia}

O presente estudo se propõe a apresentar um relato de experiência no Hospital Dr. Luiz Antonio, onde foram tratados 12 pacientes que passaram por uma anamnese, exame físico extra e intra oral, responderam a questionários específicos Portuguese Brazilian, versão 3.0 (EORTC QLQC30) e Portuguese Brazilian (EORTC QLQ-H\&N35) pré e pós 15 dias da instalação protética, comparando o antes e depois através dos testes de Wilcoxon $(\alpha=0,05)$ com objetivos exploratórios e de abordagem qualitativa (Aaronson et al., 1993; Gehan, 1965; Pereira et al., 2018). Todos os pacientes questionados assinaram o Termo de Consentimento Livre e Esclarecido permitindo a participação no estudo.

\section{Resultados}

Um total de 12 pacientes ( 9 do sexo feminino e 3 do sexo masculino) passaram por tratamento cirúrgico de câncer bucal, encaminhados pelo setor de odontologia da liga do câncer (Hospital Dr. Luiz Antônio, Natal-RN, Brasil). As aplicações dos questionários, quanto às confecções das próteses, foram realizadas na Clínica Odilon de Amorim Garcia (Universidade Potiguar - UnP, Natal - RN, Brasil), associado ao Centro Integrado da Saúde (CIS), na unidade da Salgado Filho, Natal - RN.

Os pacientes apresentavam comunicação bucosinusal, na região de palato duro e mole necessitando de prótese obturadora palatal. Os mesmos passaram por uma anamnese e um exame físico extra e intra oral em que foi verificado: análise facial, o comprometimento de dentes, tamanho e localização do defeito cirúrgico. Estes foram classificados de acordo com Okay (Figura 1) modificado em: Classe IA - palato duro, Classe IB - maxila dento-alveolar sem comprometimento de canino, Classe II - maxila dento-alveolar com comprometimento apenas de um canino, Classe III - maxila dento-alveolar com comprometimento de ambos os caninos e Classe IV- palato mole. (Okay, Genden, Buchbinder, \& Urken, 2001) 
Figura 1. Resultados da classificação de acordo com Okay. 03 pacientes apresentavam o defeito Classe IA; 02 pacientes apresentavam o defeito Classe IB; 03 pacientes apresentavam o defeito Classe II; Nenhum paciente apresentou defeito Classe III e 04 pacientes apresentavam o defeito Classe IV.

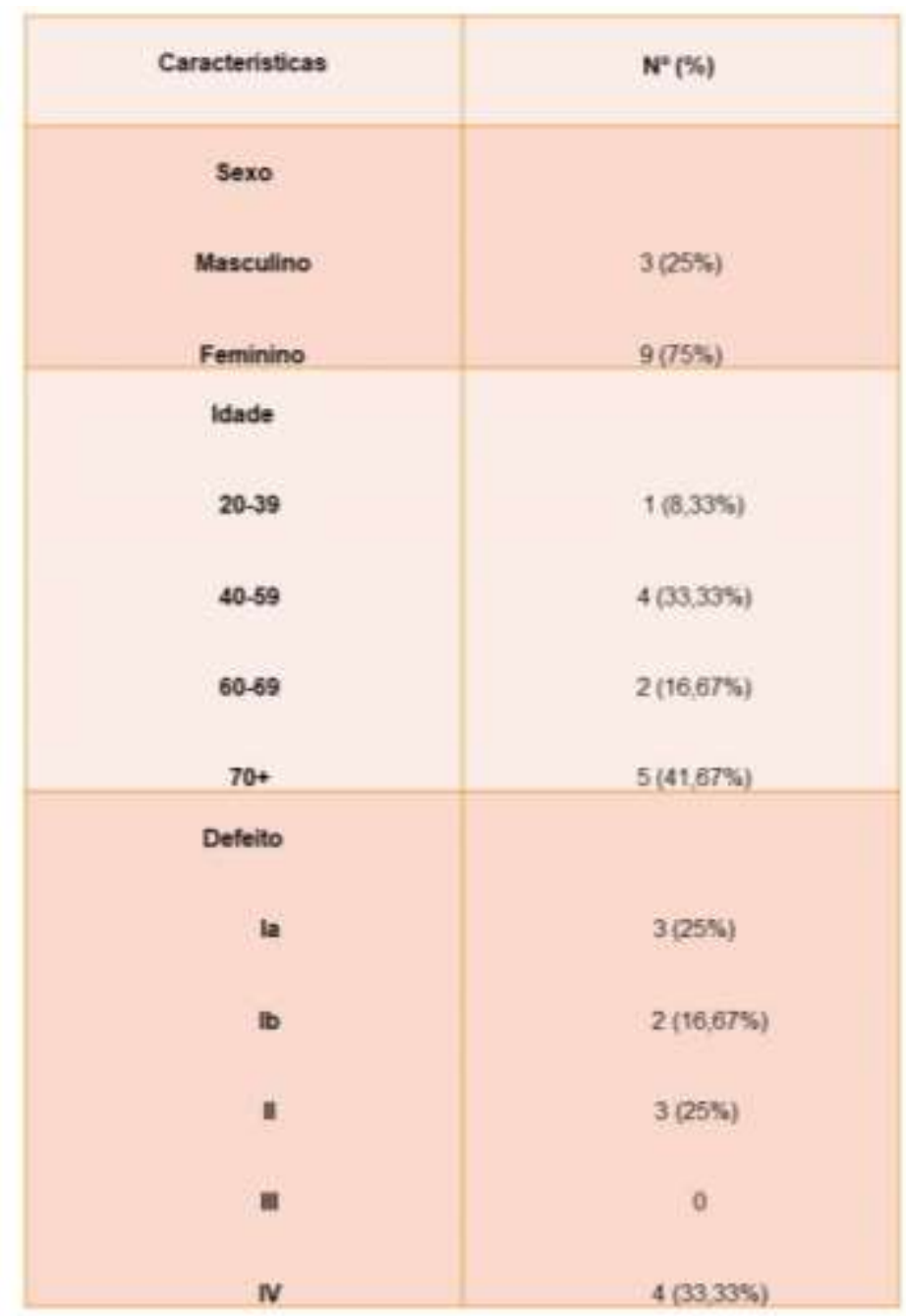

Fonte: Autores (2020).

O tratamento foi dividido em duas fases, pré e pós - protética, com 15 dias após a instalação da prótese obturadora. Foram aplicados os questionários, EORTC QLQC30_Portuguese Brazilian, versão 3.0 e EORTC QLQ-H\&N35_Portuguese Brazilian, são auto-aplicáveis e utilizados nas duas avaliações. O questionário do anexo A avalia a qualidade de vida dos pacientes com câncer de uma forma geral, observando as escalas funcionais, sintomas, estado de saúde global/qualidade de vida. A escala de sintomas é constituída dos seguintes itens: fadiga, náusea e vômito, dor, dispnéia, insônia, perda de apetite, constipação, diarréia e dificuldades financeiras. A escala funcional é composta de função física, desempenho funcional, função emocional, cognitiva e social.

O segundo questionário do anexo B é específico para pacientes com câncer de cabeça e pescoço. Nesse observa-se dor, deglutição, sentidos (tato e cheiro), fala, comendo socialmente, contato social e sexual.

Após a instalação da prótese obturadora e ajuste de adaptação com 15 dias, foi realizada a segunda aplicação dos questionários. Em todas as aplicações de questionários, os pacientes foram reavaliados clinicamente.

De acordo com o teste de Wilcoxon aplicado (Figura 2), observa-se que os melhores resultados para avaliar a qualidade de vida são obtidos de forma crescente e os da escala de sintomatologia como deglutição, comer socialmente e problemas com a fala são melhores avaliados de forma decrescente. Então, a qualidade de vida após a reabilitação com prótese 
obturadora teve melhora significativa $(\mathrm{p}=0.0090)$, assim como a deglutição $(\mathrm{p}=0.0029)$, comer socialmente $(\mathrm{p}=0.0038)$ e problemas com a fala $(\mathrm{p}=0.0131)$. Dentre todos os pacientes envolvidos na pesquisa apenas um não teve uma melhora considerável, tendo em vista que a mesma apresentava uma comunicação extensa de difícil estabilização da prótese (classe II Okay) não conseguindo se adaptar ao uso da prótese.

Figura 2. Gráfico estatístico referente à qualidade de vida, deglutição, comer socialmente e problemas com a fala dos pacientes questionados.

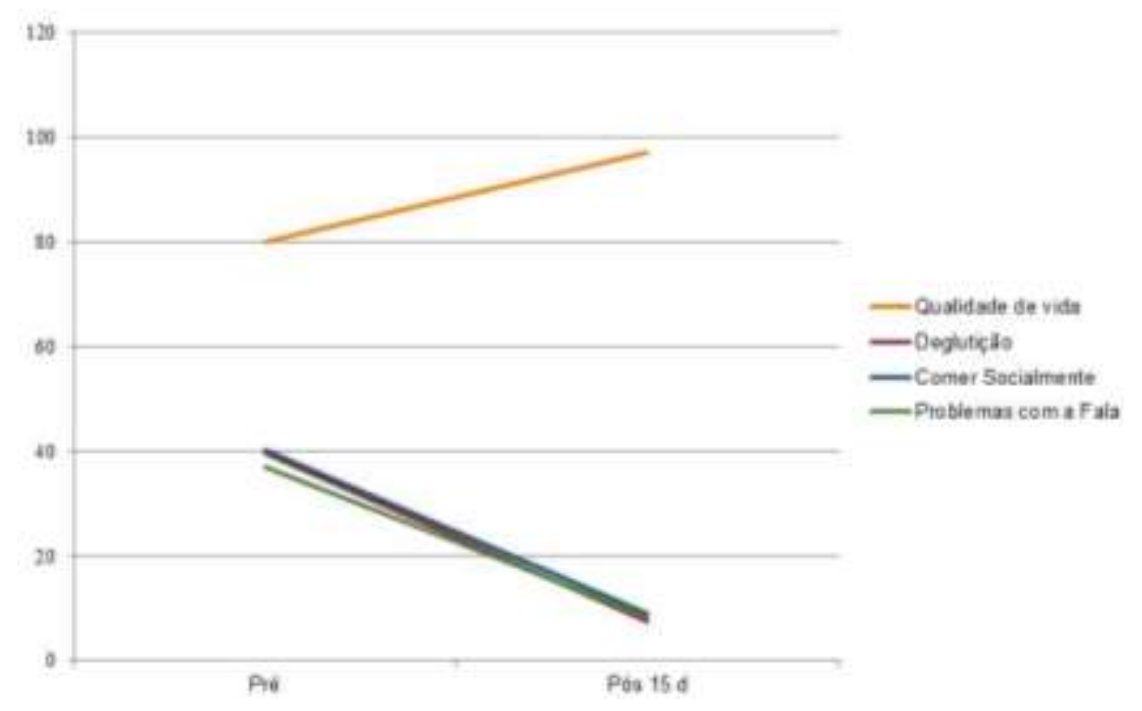

Fonte: Autores (2020).

\section{Discussão}

O impacto dos tumores maxilofaciais na vida dos pacientes são realmente expressivos não somente pelo diagnóstico do problema como também pelas sequelas após a remoção cirúrgica. Isso vem despertando a curiosidade dos estudiosos para investigar a qualidade de vida desses pacientes.

Alguns estudiosos utilizaram um Questionário de Qualidade de Vida Núcleo 30, denominado (QLQ-C30) da Organização Européia para Pesquisa e Tratamento do Câncer (EORTC) para avaliar a qualidade de vida relacionada à saúde de forma geral do paciente com câncer. Kumar et al. (2013) utilizaram um questionário mais específico para sintomatologia de cabeça e pescoço como EORTC QLQ-H \& N35. Esses dois tipos de questionários fazem parte de um sistema integrado de avaliação relacionado à saúde e qualidade de vida de pacientes com câncer que participam de ensaios clínicos internacionais. O presente estudo utilizou EORTC QLQC30_PORTUGUESE BRAZILIAN, VERSÃO 3.0, o EORTC QLQ-H \& N35. (Depprich et al., 2011; Schwarz \& Hinz, 2001; Aaronson et al., 1993)

A necessidade de um número crescente de profissionais treinados em oncologia dental é clara. Assim, há uma necessidade crescente de uma equipe de oncologia multidisciplinar formada por cirurgiões de cabeça e pescoço, oncologistas, clínicos gerais, dentistas, especialistas em oncologia e prótese maxilo-faciais, fonoaudiólogos, psicólogos e enfermeiros para educar os pacientes, garantir uma boa restauração, e criar protocolos que no futuro possam fornecer resultados funcionais e estéticos mais previsíveis. (Seignemartin, Miranda, Luz, \& Teixeira, 2015) 


\section{Conclusão}

Baseado na metodologia desenvolvida e mediante aos resultados obtidos neste relato de experiência, conclui-se que houve aumento significativo na qualidade de vida dos pacientes, redução nos problemas relacionados à deglutição, em comer socialmente e aos problemas relacionados com a fala, e a maioria dos pacientes evoluíram de forma satisfatória, tendo na fala sua remissão da hipernasalidade proporcionando uma forma inteligível.

Por fim, sugere-se também estudos clínicos controlados e randomizados que demonstrem a eficiência e eficácia da técnica de prótese obturadora.

\section{Referências}

Torres-Pereira, C. C., Angelim-Dias, A., Melo, N. S., Lemos Jr, C. A., \& Oliveira, E. M. F. D. (2012). Abordagem do câncer da boca: uma estratégia para os níveis primário e secundário de atenção em saúde. Cadernos de Saúde Pública, 28, s30-s39.

Instituto Nacional do Câncer (INCA). Estatística de Câncer. https://www.inca.gov.br/numeros-de-cancer

Elrefaey, S., Massaro, M. A., Chiocca, S., Chiesa, F., \& Ansarin, M. (2014). HPV in oropharyngeal cancer: the basics to know in clinical practice. Acta Otorhinolaryngologica Italica, 34(5), 299.

Epstein, J. B., Gorsky, M., Cabay, R. J., Day, T., \& Gonsalves, W. (2008). Screening for and diagnosis of oral premalignant lesions and oropharyngeal squamous cell carcinoma: role of primary care physicians. Canadian family physician, 54(6), 870-875.

Martínez, C., Hernández, M., Martinez, B., \& Adorno, D. (2016). Frecuencia de displasia epitelial y carcinoma escamoso en mucosa oral y orofaríngea en Chile, entre los años 1990 y 2009. Revista médica de Chile, 144(2), 169-174.

de Lima, J., Cabrera, C., Estrada Esquivel, B., Sánchez Rubio, R., \& Soler, R. (2015). Prótesis Parcial obturatriz paralelizada, en paciente oncológico. Odontoestomatología, 17(25), 42-52.

Miracca, R. A. A., Andrade Sobrinho, J. D., \& Gonçalves, A. J. (2007). Reconstrução com prótese imediata pós maxilectomia. Revista do Colégio Brasileiro de Cirurgiões, 34(5), 297-302.

Goiato, M. C., Piovezan, A. P., Santos, D. M. D., Gennari Filho, H., \& Assunção, W. G. (2006). Fatores que levam à utilização de uma prótese obturadora. Rev. Odontol. Araçatuba (Impr.), 101-106.

Aaronson, N. K., Ahmedzai, S., Bergman, B., Bullinger, M., Cull, A., Duez, N. J., \& Takeda, F. (1993). The European Organization for Research and Treatment of Cancer QLQ-C30: a quality-of-life instrument for use in international clinical trials in oncology. JNCI: Journal of the National Cancer Institute, 85(5), 365-376.

Gehan, E. A. (1965). A generalized Wilcoxon test for comparing arbitrarily singly-censored samples. Biometrika, 52(1-2), 203-224.

Pereira, A. S., Shitsuka, D. M., Parreira, F. J., \& Shitsuka, R. (2018). Metodologia da pesquisa científica. UAB/NTE/UFSM. https://repositorio. ufsm. br/bitstream/handle/1/15824/Lic_Computacao_Metodologia-Pesquisa-Cientifica. pdf.

Okay, D. J., Genden, E., Buchbinder, D., \& Urken, M. (2001). Prosthodontic guidelines for surgical reconstruction of the maxilla: a classification system of defects. The journal of prosthetic dentistry, 86(4), 352-363.

Depprich, R., Naujoks, C., Lind, D., Ommerborn, M., Meyer, U., Kübler, N. R., \& Handschel, J. (2011). Evaluation of the quality of life of patients with maxillofacial defects after prosthodontic therapy with obturator prostheses. International journal of oral and maxillofacial surgery, 40(1), 71-79.

Schwarz, R., \& Hinz, A. (2001). Reference data for the quality of life questionnaire EORTC QLQ-C30 in the general German population. European journal of cancer, 37(11), 1345-1351.

Kumar, P., Alvi, H. A., Rao, J., Singh, B. P., Jurel, S. K., Kumar, L., \& Aggarwal, H. (2013). Assessment of the quality of life in maxillectomy patients: A longitudinal study. The journal of advanced prosthodontics, 5(1), 29-35.

Seignemartin, C. P., Miranda, M. E., Luz, J. G. C., \& Teixeira, R. G. (2015). Understandability of speech predicts quality of life among maxillectomy patients restored with obturator prosthesis. Journal of Oral and Maxillofacial Surgery, 73(10), 2040-2048. 